\title{
REVIEW
}

\section{New trends in rehabilitation of children with ENT disorders}

\author{
Aggiornamenti sulla riabilitazione ORL in età pediatrica \\ R. BOVO, P. TREVISI, E. ZANOLETTI, D. CAZZADOR, T. VOLO, E. EMANUELLI, A. MARTINI \\ Department of Neuroscience, Institute of Otorhinolaryngology, University Hospital of Padua, Italy
}

\section{SUMMARY}

In the last 20 years, neonatal survival has progressively increased due to the constant amelioration of neonatal medical treatment and surgical techniques. Thus, the number of children with congenital malformations and severe chronic pathologies who need rehabilitative care has progressively increased. Rehabilitation programs for paediatric patients with disorders of voice, speech and language, communication and hearing, deglutition and breathing are not widely available in hospital settings or in long-term care facilities. In most countries, the number of physicians and technicians is still inadequate; moreover, multidisciplinary teams dedicated to paediatric patients are quite rare. The aim of the present study is to present some new trends in ENT paediatric rehabilitation.

KEY WORDS: Children $\bullet$ Rehabilitation $\bullet$ Sinusitis $\bullet$ Laryngeal paralysis $\bullet$ Choanal atresia $\bullet$ Aural atresia $\bullet$ Music $\bullet$ Voice $\bullet$ Cochlear implant

\section{RIASSUNTO}

Negli ultimi 20 anni il miglioramento dell'assistenza neonatale ha determinato un progressivo aumento dei bambini che sopravvivono in presenza di gravi malformazioni o patologie congenite. Questi bambini richiedono una riabilitazione prolungata, talora multidisciplinare e complessa. Purtroppo, un'organizzazione adeguata alla riabilitazione della disfagia, dei disturbi della comunicazione e della respirazione non è sempre disponibile, non è sempre coordinata in equipe multidisciplinari che operino sia negli ospedali che sul territorio e non è facile mantenere tutte le figure professionali coinvolte al passo con le sempre più rapide innovazioni. Scopo del presente lavoro è presentare un aggiornamento su alcuni aspetti tuttora controversi della riabilitazione in età pediatrica.

PAROLE CHIAVE: Bambini $\bullet$ Riabilitazione $\bullet$ Sinusite $\bullet$ Paralisi laringea $\bullet$ Atresia coanale $\bullet$ Atresia auris $\bullet$ Musica $\bullet$ Voce $\bullet$ Impianto cocleare

\section{Introduction}

In the last 20 years, neonatal survival has progressively increased due to the constant amelioration of neonatal medical treatment and surgical techniques. These aspects have produced an increased incidence of children with congenital malformations and severe chronic pathologies. Moreover, the immigration of children from underdeveloped countries, where perinatal infections or other risk factors are still high, have further increased the number of paediatric patients who need rehabilitative care. It is worth noting that among over 50,000 scientific papers in the literature regarding paediatric rehabilitation, only about 1000 are related to ENT arguments. Thus, rehabilitation of paediatric patients with disorders of voice, speech and language, communication and hearing, deglutition and breathing is generally still inadequate to the real needs and rarely is well organised with multidisciplinary teams, working both in the hospital setting and in long-term care facilities. The aim of the present study is to present some new trends in ENT paediatric rehabilitation.

\section{Chronic rhinosinusitis}

Rhinosinusitis is a very common condition and its prevalence has increased in recent years in both children and the adult population. By definition in chronic rhinosinusitis (CRS), symptoms last more than 12 weeks. Antibiotics are the most frequently used therapeutic agents in acute rhinosinusitis (ARS). Uncomplicated ARS, if no allergies exist, can be treated with amoxicillin $(40 \mathrm{mg} / \mathrm{kg} /$ day or $80 \mathrm{mg} / \mathrm{kg} / \mathrm{day}$ ). Other reasonable choices are amoxicillin/ clavulanate and cephalosporins. If hypersensitivity to any of the above antimicrobials is suspected, alternative choices include trimethoprim/sulfamethoxasole, azithromycin, or clarithromycin ${ }^{1}$. Surgical intervention in the treatment of nasal polyps is considered in patients who fail to improve after a trial of maximal medical treatment or in patients presented at diagnosis with antrochoanal polyp, cystic fibrosis (CF), Kartagener and Churg-Strauss syndromes. Functional endoscopic sinus surgery (FESS) involves the clearance of polyps and polypoid mucosa and opening of the sinuses ostia. If maximum medical therapy is unsuccessful in a 
child with chronic or recurrent sinusitis, evaluation for underlying medical disorders (e.g. immunodeficiency, allergic rhinitis, CF and immotile cilia syndrome) is warranted. Surgery consisted of adenoidectomy with or without antral irrigation and balloon sinus dilation, and FESS ${ }^{2}$.

Follow-up begins after surgery and can last for years; it is performed with nasal endoscopy and inspection of breathing spaces, eventual nasal secretions, healing of nasal mucosa, natural ostium and sinusotomy patency. In the first visit after FESS, there is removal of nasal packing; it is normally performed one or two days after surgery. Further visits are made at 15 days and one month after surgery: the goal is inspection of nasal cavities and removal of nasal crusting and fibrinous exudate to prevent nasal adhesions. If no complications exist, clinical follow-up visits are scheduled 3-6 and 12 months after surgery and are made to prevent late complications and relapse. Recurrence rates are higher in nasal polyposis stage IV and V (association with CF, Kartagener and Churg-Strauss syndrome). In these cases, there is no definitive treatment for nasal polyposis and long follow-up is necessary. In the youngest patients, endoscopic medications must be scheduled in sedation or under general anaesthesia: parents should be previously informed about this requirement and the fact that these are not new surgical interventions, but are necessary to improve the first result. All children with CRS should be submitted to allergy tests. Tests for immunodeficiency should be done in children with chronic recurrent disease, poor response to medical treatment, a history of other chronic infections (such as recurrent pneumonia or otitis) or when unusual microorganisms are isolated in the nasal secretion. To obtain the best results, surgery in children should be performed by expert surgeon after a skills trial in phlogistic, malformative and neoplastic diseases in adults and using surgical instruments dedicated to the paediatric population.

The health impact of chronic recurrent rhinosinusitis of paediatric patients and their parents is severe. Children with rhinosinusitis are perceived by their parents to have significantly more bodily pain and to be more limited on physical activities than children with asthma, juvenille rheumatoid arthritis and other chronic disorders ${ }^{3}$. The $\mathrm{SN}-5$ is a validated symptom score questionnaire for the evaluation of CRS in children ${ }^{4}$. The self-administered survey is completed by the child's parent using 7-point ordinal response scales for each item. Domains include sinus infection, nasal obstruction, allergy symptoms, medication use, emotional distress and activity limitations.

\section{Choanal atresia rehabilitation in paediatric patients}

Choanal atresia is defined as a congenital obstruction of the posterior nasal choanae. It represents one of the most frequently observed congenital nasal defects, with a prevalence of 1:5,000-8,000 live births ${ }^{5}$. In about $50 \%$ of cases it occurs bilaterally, thus constituting the main indication for sinonasal surgery in the newborn ${ }^{6}$.

The association of choanal atresia with craniofacial and genetic syndromes is well known. In particular, between $7 \%$ and $29 \%$ of patients with choanal atresia are affected by CHARGE syndrome. In these conditions, the atresia presents often bilaterally (Fig. 1) ${ }^{5}$.

Bilateral choanal atresia presents with respiratory distress in the immediate neonatal period. The crises are typically alleviated when the child begins to cry. Surgical intervention is considered the main therapeutic option. Over the decades, various surgical techniques have been described ${ }^{7-9}$.

An "ideal" surgical technique should ensure adequate choanal patency and low restenosis rates, should spare the surrounding anatomical structures and provide low rates of morbidity and mortality. It should also ensure the shortest time hospitalisation ${ }^{8}$.

Nowadays there is still a lack of randomised controlled studies comparing the efficacy of the different surgical approaches, but it is common expert opinion to consider an endonasal endoscopic approach to be the least invasive and probably the safest in terms of compliance ${ }^{9}$.

However, restenosis remains the most frequent complication in choanal atresia surgery, with a prevalence between $9 \%$ and $36 \%$. Adjuvant measures have been introduced to reduce the restenosis rate: nasal stenting, mitomycin $\mathrm{C}$ and balloon dilatation. The use of nasal stenting is still debated ${ }^{10}$.

A stent is generally applied to stabilise postoperative outcomes, but this procedure entails some worrisome complications. Nasal and nasopharyngeal infections, mucosal ulcerations, tissue ischaemia and necrosis and formation of granulation tissue are only some of the possible consequences of long-term use of a nasal stent ${ }^{10}{ }^{11}$.

Recently, some authors performed stentless choanal endoscopic surgery repair. To date, a total of 42 procedures have been carried out, with a restenosis rate of only $38 \%{ }^{11-13}$. Nasal irrigation in the postoperative period is crucial for maintaining neo-choanal patency, in association to endoscopic medications according to a precise follow-up schema. In our centre, nasal packing is removed 24 hours after the surgical procedure, the first postopera-

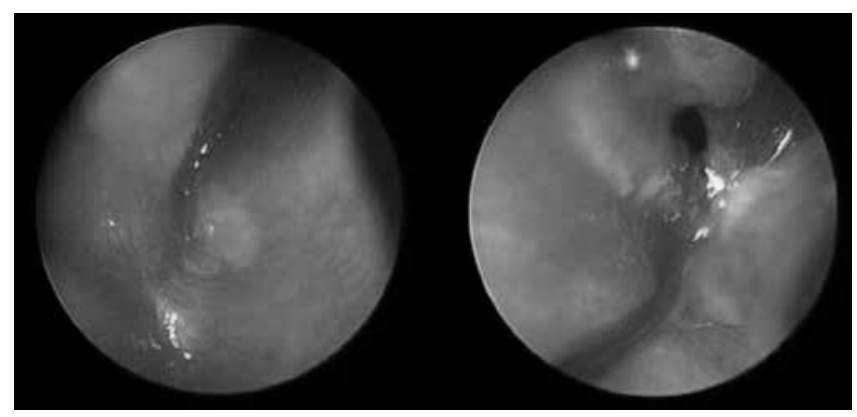

Fig. 1. Endoscopic view of a bilateral choanal atresia. 
tive visit is generally performed after 7 days, under sedation. Further visits take place after 15, 45 and 90 days, and then at 6 and 12 months.

Anti-reflux agents are helpful in avoiding granulation tissue formation, since gastro-oesophageal reflux is a negative predictive factor for restenosis, with incorrect postoperative management and an early age at surgery ( $>10$ years) ${ }^{14}$.

\section{Obstructive sleep apnoea}

Sleep disorders include different conditions ranging from primary snoring to obstructive sleep apnoea (OSA). OSA is characterised by both partial and complete airway obstruction, which induce hypoxaemia with a relevant decrease in quality of the sleep and day life.

OSA in children has a prevalence between $1.1 \%$ and $2.9 \%{ }^{15}$. The diagnostic gold standard in OSA syndrome is overnight polysomnography, but home pulse oximetry has also been proposed as a screening test ${ }^{16}$. Adenotonsillar hypertrophy is the most important aetiologic factor for OSA in paediatric patients: nearly $45 \%$ of OSA children are affected by lymphatic Waldeyer's ring hypertrophy ${ }^{17}$. Adeno-tonsillectomy is therefore indicated as a first therapeutic option in OSA children without other important comorbidities and has shown good efficacy in improving the patients' quality of life ${ }^{18}$. However, the degree of adenotonsillar hypertrophy is not strictly related to the severity of respiratory sleep disorder. Predictors for a persistent elevated postoperative apnoea-hypopnoea index (AHI) are: age $>7$ years, $\mathrm{BMI}$ increase, preoperative $\mathrm{AHI}>20$ and asthma.

As reported by Albera et al. in a large cohort of paediatric patients, more OSA children presented with impaired swallowing function and impaired speech articulation in comparison to non-OSA patients. Interestingly, adeno-tonsillectomy improved obstructive respiratory function in the first group of patients, but did not modify secondary dysfunctions such as atypical swallowing, oral breathing, or dyslalia ${ }^{19}$.

A rehabilitative programme is necessary in addition to surgical or medical therapy for OSA. No standardised protocols are reported to date ${ }^{20}$. The speech therapy centre in Rome has proposed a valid rehabilitation scheme, which consists of neuromuscular and myofunctional therapy for oro-facial musculature ${ }^{21}$.

\section{Laryngeal paralysis}

Laryngeal paralysis (LP) is the second most common laryngeal congenital malformation after laryngomalacia. Of all laryngeal malformations, its prevalence is about 15-20\%. Unilateral LP (48\%) may cause swallowing impairments and chronic food inhalation; depending on the position of fixation of the vocal cords, bilateral LP (52\%) may present with respiratory symptoms varying from inspiratory stridor to acute respiratory distress with cyanosis. The latter condition requires emergent tracheal intubation ${ }^{22}$.
A flexible fiberscope is routinely used in the diagnostic workup of LP. It is performed without anesthesia, thus allowing direct visualisation of laryngeal motility. Possible underlying associated malformation, which is present in about $45 \%$ of LP cases, is investigated with a rigid laryngo-tracheo-bronchoscope under general anaesthesia. Only after both central and peripheral causes of vocal fold paralysis have been excluded, the condition can be defined as idiopathic. In severe cases of LP, when the risk of liquid inhalation occurs, it is necessary to place a nasal-feeding tube. In addition, tracheostomy is required in $8 \%$ and 53\% of cases of unilateral and bilateral LP, respectively ${ }^{22}$.

Above all, idiopathic LP may partially spontaneously resolve within the $6^{\text {th }}-12^{\text {th }}$ month of life. When there is no evidence of improvement in laryngeal motility after the age of 2 years, the lesion could be defined persistent. At this point, surgical intervention is required to create adequate respiratory space. A posterior cordectomy is generally performed ${ }^{23}$.

Logopaedic rehabilitation (LR) is indicated in unilateral LP with the aim of inducing a spontaneous compensation of vocal fold motility. The earlier LR is begun, the better the functional results that might be obtained. Moreover, LR finds application in children who underwent tracheal tube or nasal-feeding tube placement. Long lasting tracheal intubation ( $>48 \mathrm{~h}$ ), as well as nasal-feeding tube application, can provoke vocal and swallowing dysfunctions when removed. Tracheostomy, moreover, affects laryngeal motility in indirect ways, determining deafferentation of the proprioceptive innervation in the transglottic area and progressive muscular atrophy ${ }^{24}$. A LR programme is defined on the basis of symptoms. Plurisensorial stimulation touches all the structures involved in respiratory, vocal and deglutition functions.

\section{Juvenille laryngeal papillomatosis}

Juvenille laryngeal recurrent papillomatosis (JRRP) is a rare viral disease caused by HPV-6 and HPV-11 infection. Both single and multiple lesions of JRRP affect especially the glottic region but, in 5-28\% of cases, may also extend caudally into the tracheobronchial three and lungs ${ }^{25}$. The papillomatosis glottic involvement entails important functional and vocal consequences, with significant impact on the emotional and behavioural sphere of children, and consequently their quality of life.

Although in JRRP the goal of treatment is obtaining adequate respiratory airway patency to avoid tracheostomy, it is also of utmost importance to define suitable surgical treatment protocols to reduce the incidence of iatrogenic vocal damage.

The intensity of voice function alteration correlates with the number of surgical procedures per person, and not with age at diagnosis ${ }^{26}$. Consequently, voice function preservation surgery should preserve as much healthy la- 
ryngeal mucosal tissue as possible, without reaching radical excision.

The gold standard treatment is a mini-invasive approach with $\mathrm{CO}_{2}$ laser or microdebrider surgical excision of lesions, associated or not to adjuvant virostatics ${ }^{27}$. Only one prospective cohort study in 11 patients compared vocal function outcomes of the two surgical techniques. Main vocal outcomes included overall severity rating, jitter, shimmer and noise-toharmonic ratio. "Cold" dissection with a microdebrider resulted in better immediate and early postoperative voice outcomes in children. Additionally, increased exposure to $\mathrm{CO}_{2}$ laser correlated with worsening voice quality. This can be explained by the potential thermal injuries of the surrounding and deeper tissues induced by the laser ${ }^{28}$.

Long-term soft tissue complications including scarring, stenosis and web formation can also lead to voice disorders. The total number of repeated microsurgical interventions performed per patient and the lesion site influences the rate of these complications. The most frequent laserinduced soft tissue complication is anterior glottic web ${ }^{25}$. The rate of soft tissue complications is influenced not only by the total number of repeated surgical interventions per patient, lesion site, surgical technique, age at diagnosis and advanced stage of disease, but it also correlates with the presence of gastro-oesophageal reflux ${ }^{29}$.

The main functional aim in JRRP surgery is therefore avoiding soft tissue deep laryngeal damage in order to prevent voice function injuries in children. A "cold" dissection of the glottic lesions sparing the lamina propria, healthy surrounding tissue and glottal deeper laryngeal structures seems to be the right direction. The use of a microdebrider is reaching increasing consent in this field. However, in literature there are no prospective randomised studies to support the superiority of the microdebrider surgical technique. In the future, the use of tetravalent HPV vaccine might represent a promising option.

\section{Breathing rehabilitation in patients with laryngo-tracheal axis stenosis}

The most frequent diseases causing laryngo-tracheal stenosis in the paediatric population and requiring breathing rehabilitation are: laryngomalacia, vocal cord paralysis (monolateral or bilateral), hypoglottic stenosis, tracheal stenosis and tracheomalacia.

Laryngomalacia is a congenital abnormality of the laryngeal cartilage. It is a dynamic lesion resulting in collapse of the supraglottic structures during inspiration, leading to airway obstruction. It is thought to represent a delay of maturation of the supporting structures of the larynx ${ }^{30}$. If present, reflux can worse breath symptoms ${ }^{31}$. Laryngomalacia is the most common cause of congenital stridor and is the most common congenital lesion of the larynx. Diagnosis is made by flexible naso-pharyngo-laryngoscope under topical or no anaesthesia, followed by direct laryn- goscopy under general anaesthesia (without muscle relaxant). In order to complete the staging, pulmonary function tests (PFTs) and polysomnography (PSG) are mandatory ${ }^{31}$. Spontaneous resolution of symptoms is the rule; it usually occurs by the age of 2-5 years, so the main treatment consists in antireflux drugs and follow-up. Surgical intervention is indicated only in severe cases (10-12\% of all cases) of laryngomalacia and consists in endoscopic approaches, namely supraglottoplasty. The aim of surgery is to correct the anatomical cause with minimal tissue damage. A cold steel technique is recommended. Other instruments have been used including laser and microdebriders.

In recent years, paediatric breathing physiotherapy uses various techniques to help removal of mucus from the airways and improvement of pulmonary function. These are: cough, compressions/vibrations, forced expiratory technique (FET), autogenic drainage (AD), prolonged slow expiration (PSE), positive expiratory pressure (PEP), positive continuous periodic pressure (PCPAP), continuous positive airway pressure (CPAP), physical exercise, aerosol and nasal unblocking.

Evaluation of patient posture is another important issue: chest conformation, postural alignment (chin, shoulder, thorax position) and spine alignment on frontal and sagittal plane. The main goal of breathing treatment is removal of airway secretions, where physiological clearance mechanisms (cough, cilia, ventilation) are ineffective.

\section{Paediatric tracheostomy: a changing trend}

In the last 30 years, the role and the indications for tracheostomy have remarkably changed. The most common indications for paediatric tracheostomy have passed from infective causes (epiglottitis, croup, diphtheria) to airway obstruction and anomalies ${ }^{32}$, long-term ventilation requirement and underlying neuromuscular or respiratory problems ${ }^{33}$.

Long-term intubation is now the first indication for tracheostomy; in the absence of guidelines, the majority of authors agree to perform a tracheostomy after 2-3 weeks of intubation to avoid hypoglottic or tracheal stenosis ${ }^{34}$. A literature review shows that $50 \%$ of patients with tracheostomy is less than one year old. This is related to the improvement of the Paediatric Intensive Care Unit (PICU) that allows more premature babies survive, but at the same time it entails greater risk of complications for the smaller diameter and the lower stiffness of the trachea. More generally, there has been in recent years an increase of late complications of trachestomy, related to the prolonged presence of the tracheostomy tube.

These late complications are peristomal granulomas, tracheal stenosis, trachea-innominate fistula, trachea-oesophageal fistula and trachea-cutaneous fistula.

The surgical procedure is more complicated in the paediatric population. Open surgical tracheostomy and percutaneous dilatational tracheostomy, described by Ciaglia in $1985^{35}$, are two possible approaches in the adult population. 
There are different types of tracheostomy tubes that vary in certain features for different purposes; they can be made in plastic, silicon, PVC and metal. For the first application, it is generally suggested to use a plastic tube. The length and angle of the tube should be such as to maintain its end portion in axis with the trachea. The American Thoracic Society suggests selecting a tube of such a length as to extend to $2 \mathrm{~cm}$ below the stoma and at least $1-2 \mathrm{~cm}$ from the carina ${ }^{36}$. The National Guideline Clearinghouse (NGC) ${ }^{37}$ recommend that skin care of the stoma and under the tracheostomy ties be provided at least daily, and more often if indicated, to prevent pressure necrosis and to maintain intact, clean and dry skin. Tracheostomy tube suctioning should be performed at least twice daily and as needed, based on clinical assessment to assure tracheostomy tube patency (ATS). It is recommended that tracheostomy tube changes are performed routinely by institutional standards to maintain airway patency; the first change is made within 5-7 days for surgical tracheostomy and within 10-14 days for percutaneous tracheostomy. The further changes are routinely made every 2-4 weeks according to the Cincinnati Children's Hospital Center (CCHMC) guidelines ${ }^{38}$. There is insufficient evidence and a lack of consensus to make a recommendation on the use of heated versus cool humidification in prevention of mucous plugging (ATS). According to AAO-HNSF decannulation can be made if clinically there is resolution of the primary disease, no active infection, tolerance of the speaking valve, endoscopically there is a clear tracheobronchial tree without suprastomal granuloma and functionally if there is an adequate cough reflex. The process of decannulation needs observation for 24-48 in a monitored setting ${ }^{36}$. Every child with tracheostomy should be referred to a phoniatric clinic for evaluation of the phonation and deglutition. Rehabilitation of speech relies on phonatory valves, signs language, laryngophone and fenestrated tracheotomy tubes. Evaluation of the deglutition to rehabilitate the patient for feeding is made with administration of methylene blue solution under videolaryngoscopy and with scintigraphic study of deglutition.

\section{Dysphagia following laryngo-tracheal reconstruction}

The laryngeal anatomical structures involved in swallowing are epiglottis, laryngeal vestibule and vocal folds ${ }^{39} 40$. Anatomical alterations of upper airways causing stenosis may prevent coordinated process of swallowing, affecting its efficiency and safety ${ }^{41}$. Surgery designed to restore the airway in laryngo-tracheal anomalies can cause or exacerbate the alterations of swallowing mechanisms and interfere transiently or permanently on airway protection mechanisms causing dysphagia. Surgery of the airway include both endoscopic (epiglottoplasty, dilatations and balloon surgery, vocal fold lateralisation, partial arytenoidectomy, closure of laryngo-tracheo-esophageal cleft, posterior cordotomy) and open surgery (laryngotracheo- plasty for airway dilatation, laryngotracheal reconstructions, cricotracheal resection with removal of stenotic tract and termino-terminal anastomosis).

The difficulties in feeding and swallowing after laryngo-tracheal reconstruction are multifactorial and to be able to make a rehabilitative project is mandatory understand the reason of these difficulties. There are specific and non-specific factors that contribute to the disease. Non-specific factors are basic diagnosis, health status of the baby and type of surgery which has undergone. Specific factors can be further divided in immediate and delayed. The immediate factors are anatomical structure transformation, altered protection of airway and presence or absence of the tracheostomal tube. The delayed factors are development of sensory skills, acceptance of feeding aids, acceptance of food more consistent, development of oral motor skills like chewing, adjusting meal times and appropriate eating behaviour.

Considering the importance of swallowing and breathing for general health and in the quality of life of the child, it is very important to manage these diseases with a multidisciplinary approach involving surgical, medical and rehabilitative features ${ }^{42}$.

In the literature, there are many studies that stress the importance of evaluating feeding and swallowing skills of the child before and after surgery; this is done to better understand the functional ability of the child and understand if the swallowing problems are related to the basic diagnosis or a consequence of the surgery on anatomical structures ${ }^{43-45}$. The evaluation protocol includes the presence of alternative nutrition; evaluation of the different mechanisms of sensory and motor parts of deglutition; assessment of the management of oral secretions; the evaluation of the mechanisms of sucking/chewing and swallowing; breathing during the meal; and the amount of food administered. If swallowing function will be evaluated with instrumental tests, the child may undergo a video-fluoroscopic swallowing exam.

The goal of preoperative evaluation is to understand the anatomical and physiological factors that determine any difficulty feeding in order to react appropriately and quickly in the postoperative period. After intervention, assessment will be carried out to evaluate the results and make a rehabilitation plan with the objective of recovering the best features. The rehabilitation project must be customised and targeted, and should not only take into account swallowing function, but also respiratory function, and must include weaning from tracheal cannula if de-cannulation is possible, or use of speech valve.

\section{Voice rehabilitation in children: two different possible protocols}

The Voice Craft method (Estill Voice Training) was established in 1988 by American singing voice specialist Jo Estill and is characterised by a series of vocal manoeuvers to develop specific control over individual muscle 
groups within the vocal mechanism ${ }^{46}$. Training comprises 13 vocal exercises or compulsory figures. Each 'figure' establishes control over a specific structure of the vocal mechanism, in isolation, by moving the structure through a number of positions. The most important compulsory figure is probably the false vocal folds control, which identifies three possible positions of the false vocal folds: constricted, mid and retracted. Moreover, Estill Voice Training incorporates six 'voice qualities' as mechanisms for demonstration of voice production control. The six voice qualities are speech, sob, twang, opera, belting and falsetto. A protocol of 12 training sessions for the voice rehabilitation in children has been described by Fussi and Turlà ${ }^{47}$. In the first session, children and parents cooperate in collecting clinical history and receiving together initial and simple counseling about vocal hygiene and possible consequences of voice disorders. Children receive a diary to note correct or un-correct vocal use during the day every evening. During the second session, several relaxing and stretching exercises are carried out. Perceptive training (starting from the intensity parameter) is taught during the third session, using specific games and exercises. The fourth and fifth sessions are dedicated to correct breathing and pneumo-phonic coordination. These aspects are generally boring to children and need to be taught through different games (soap balls, wind imitation, etc.). The sixth session is dedicated to control of the correct acquisition of breathing and to further auditory perception training on intensity and pitch. In the seventh session exercises of articulation and exercises to experiment and perceive vocal tract resonance are proposed. Several nasalisation and chewing movements are explained. During the eighth session, children are invited to sing a simple tune using different modalities of voice productions, correct breathing and coordination, hyperarticulation etc. The basic vocal qualities described in the Estill method are explained in the ninth lesson: speech, sob, belting and twang. During the $10^{\text {th }}$ and $11^{\text {th }}$ lessons, children must produce the different voice qualities by imitating several cartoon characters. Finally, during the last session participants are involved in dramatising a simple story by using different voice qualities and productions, depending on the specific context of the story. Post-treatment evaluation generally demonstrates that children have acquired better consciousness of their voice instrument, use different voice modalities depending on the specific situation, significantly reduce vocal abuse and enjoy in playing with their voice.

The aim of the "Proprioceptive-Elastic Voice Rehabilitation Program" (PROEL) (Fig. 2) ${ }^{48}$ is to achieve a relaxation of muscle stiffness and to obtain elasticity through facilitating postures, unstable balance, and body movement. The method of work is "experimental": a hoarse patient compares his/her voice before and after exercise, and thus makes an "impression" on the brain that allows them to internalise a "phonatory pattern" correctly. Perception

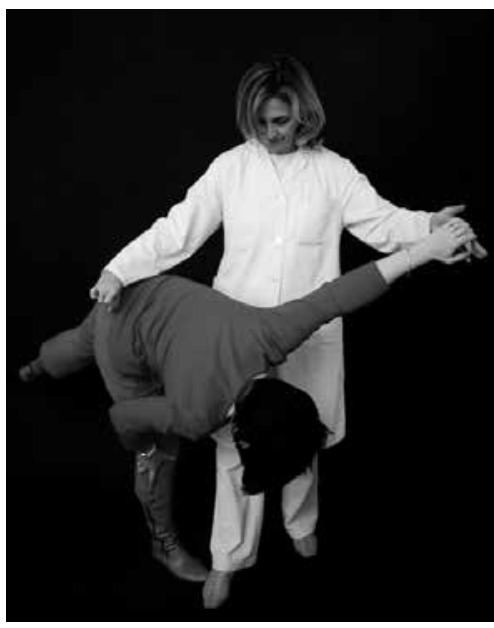

Fig. 2. Proprioceptive-elastic therapy (PROEL).

training is thus fundamental even in the PROEL method: children should become conscious of their voice qualities, of the mechanisms of voice production, how to use the voice in the different daily situations and how to avoid voice fatigue. Rehabilitative training should be considered completed when the child has perfectly consolidated the correct use of voice in relation to total body, is able to recognise the possible risk factors for his/her voice and is able to self-correct voice misuse or abuse without an external guide. The PROEL method has been modified by De Maio et al. ${ }^{49}$ for optimal use in paediatric dysphonic patients. Ludic activities, on several occasions chosen by the young participants themselves, have demonstrated to be useful in improving voice production and enjoyable by the children.

A new instrument for the evaluation of the benefits obtained with different treatments for the voice disorders in children is the Children Voice Handicap Index 10 questionnaire (CVHI-10). This questionnaire is easily administered, highly reproducible, with good clinical validity and responsiveness to treatment ${ }^{50}$.

\section{Aural atresia rehabilitation}

Patients with bilateral atresia should have either a softband BAHI (Bone Anchored Hearing Implant) or a conventional headband bone conduction hearing aid in early life to provide adequate stimulation for development of the central nervous system. When the child grows older, percutaneous BAHI becomes an option ${ }^{51}$, usually by 5 years old. The surgical technique has been recently simplified with the linear incision (Fig. 3), instead of the skin flap technique ${ }^{52}$.

Canalplasty is a choice for selected candidates after the age of 8 . The anatomy of the temporal bone is a major confounding factor in the approach of hearing rehabilitation. Patients with good middle ear anatomy (Jahrsdoerfer score 7 or above) ${ }^{53}$ may be suitable for canal reconstructions, although this kind of surgery is less frequently carried out than in the past. In most studies comparing BAHI and patients with EAC recon- 


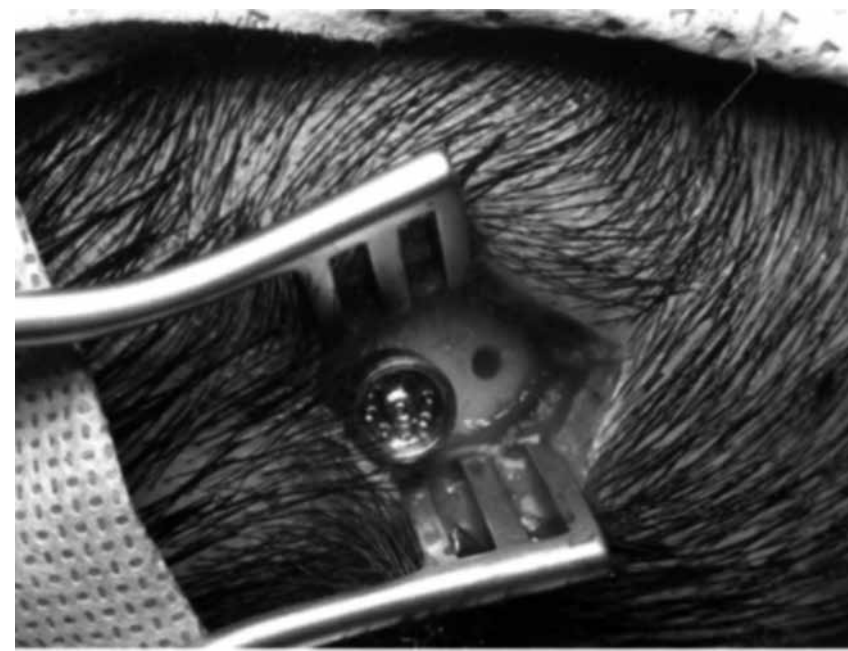

Fig. 3. The linear incision and the insertion of a new-profiled abutment.

struction, BAHI has generally resulted in significant hearing gain vs. reconstruction ${ }^{54}$. The mean postoperative speech reception threshold is $25-35 \mathrm{~dB} \mathrm{HL}$, which is the range of mild hearing loss and around $30 \%$ of patients still need a conventional hearing aid to assist with hearing after surgery ${ }^{54}$. In addition, otologists are often frustrated with the relatively common occurrence of restenosis and recurrent infections of the canal (Fig. 4). The reported rate of restenosis ranges from 5 to $29 \%$ and around $26 \%$ of the population requires reoperation. Serious complications may also occur during this surgery, such as worsening of hearing loss and facial nerve palsy in $1 \%$ of cases, as up to $30 \%$ of this group of patients have an anomaly in the course of their facial nerve ${ }^{5556}$.

Vibrant sound bridge (VSB), on the other hand, is implanted inside one middle ear, which provides unilateral stimulation to the inner ear system. This unilateral direct inner ear stimulation completely removes the possibility of signal confusion. VSB is a middle ear implant consisting of two parts, the external audio processor (EAP) and the implantable vibrating ossicular prosthesis (VORP). The EAP picks up sound signals, amplifies them and transmits them to the VORP. The floating mass transducer (FMT), in the distal part of the VORP, vibrates the attached middle ear structure through a single point of attachment and thereby stimulates the cochlea.

In aural atresia patients, the FMT can either be attached to the stapes, if it is functioning, or to the round window, in which case the procedure is known as round window vibroplasty. During VSB implantation, the usually malformed middle ear cleft has to be entered to insert the FMT. It carries a risk of injury to facial nerves and the inner ear, while no such complications occur in BAHI.

Bonebridge (BB) was initially designed for patients over 18 years old because a certain thickness of the cortical bone is required. Despite some reports of successfully im-

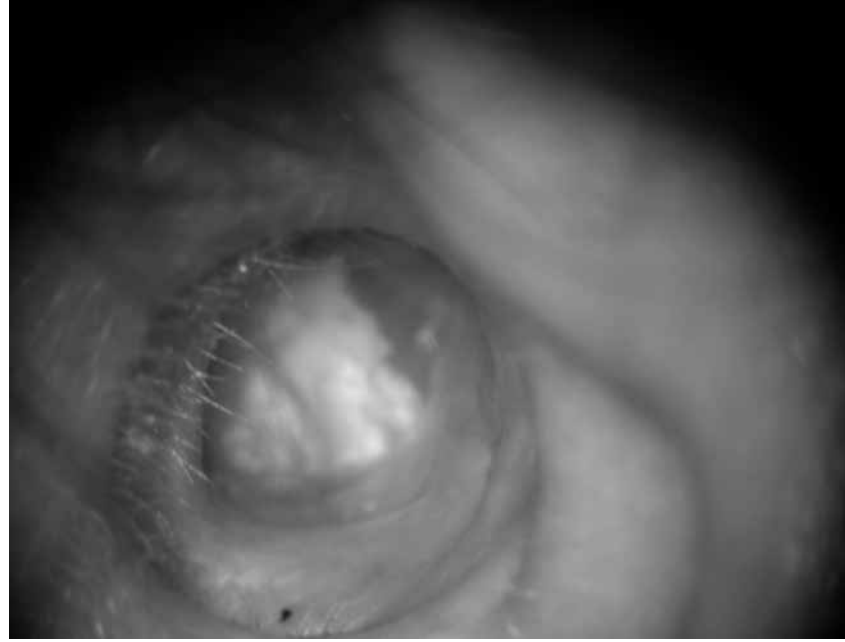

Fig. 4. Apparent good surgical outcomes of atresiaplasty. However, recurrent external otitis exacerbated by whatever kind of ear moulds required the implantation of BAHI.

planted paediatric patients, this procedure requires a great deal of caution.

For those with unilateral disease, time is allowed for a thorough discussion and consideration of treatment options. In fact, it has been well established that patients with unilateral hearing loss may have difficulties in communication and at least $25 \%$ of the patients' parents and teachers report behavioural problems and academic performance issues ${ }^{57}$.

There is still controversy about how much we should do for patients with unilateral congenital aural atresia. Restoring binaural hearing can avoid the development of an ear dominance syndrome and numerous studies have shown that it brings more benefits than harm, including better hearing in noise, improved distance hearing and elimination of headshadowing ${ }^{58}$. However, to avoid a dominant syndrome, binaural hearing should be promptly restored, most probably in the first years of life. As every patient's needs and expectations vary, detailed discussion should take place to achieve the best solution for each individual case.

In some patients who need repeated magnetic resonance imaging (MRI), for example in patients with neurological diseases, the method of hearing rehabilitation has to be carefully considered. This is because MRI is generally not recommended for patients with implantable hearing aids, like VSB. BB can tolerate MRI scanning up to 1.5 tesla and BAHI up to 3 Tesla. These implants also produce artifacts in images of the brain. BAHI is the least disturbing due to its small implant size.

In summary, most patients with aural atresia benefit from hearing rehabilitation. The choices are conventional headband bone conduction hearing aid, softband BAHI, canalplasty, percutaneous BAHI, VSB and BB. Each option has its strengths and weaknesses. Early identification and referral for further management are key to obtain a successful long-term outcome. 


\section{Cochlear implant}

Cochlear implant in children is a safe procedure with a low risk of complications as largely reported in recent literature $^{59-61}$. Immediate and late complications are not significantly different when surgery is carried out before one year of age, with respect to $12-18$ months $(P=1)$. Moreover, anaesthesiological complications are rare, probably ranging from 0 to $1.5 \%$, even in syndromic patients ${ }^{62}$. Early implantation is believed to enable the maximal development of communication skills, social exchange and cognitive abilities. The lower age limit of implantation is therefore not related to surgical or anaesthesiological considerations, but to the consideration that very early audiological diagnosis is not always reliable, even when established at tertiary centres, and not all children identified as suffering from sensorineural hearing loss (SNHL) will have permanently impaired threshold levels. The absence of any electrophysiological or behavioural reaction to sound does not always reflect SNHL. Bovo et al. ${ }^{61}$ have recently described a case series of 23 newborns with a diagnosis of severe-to-profound hearing loss at three months, who significantly improved even reaching a normal auditory threshold during the first year of life. All the full term neonates showed a significant improvement in their initial threshold within 6 months of age. On the other hand, in most of the premature newborns the initial signs of threshold amelioration occurred beyond 70 weeks of gestational age, and even beyond 85 weeks in one case. The authors assumed that when severe-to-profound SNHL is confirmed and persistent after appropriate follow-up tests for 6-8 months, cochlear implantation can be carried out with no risk of diagnostic error in term-born infants. On the contrary, follow-up for severely pre-term babies should last up until no less than 80 weeks of gestational age. The lower limit of age must not be respected in all cases of post-meningitic deafness or in other causes of cochlear fibrosis and calcification.

\section{Music rehabilitation in children with hearing aid and cochlear implant}

According to Ford ${ }^{63}$, "the capacity to perceive and assimilate music resides in the brain, and although hearing loss may impose certain limitations upon the extent to which musical potential is realised, it does not negate the presence of innate musicality". Furthermore, Darrow ${ }^{64}$ stated that "for the deaf or hard of hearing children, music can be in some ways more aurally accessible than speech". There is today a general agreement that these children can enjoy and participate in music activities and can increase their aesthetic sensitivity ${ }^{65-67}$.

Nevertheless, it is to be considered that among the three cardinal elements of music, i.e. rhythm, melody and timbre, in most hearing aided or implanted deaf children only rhythm perception is reported to be similar to that of listeners with normal hearing.

In fact, even with technically sophisticated multiple-channel sound processors, recognition of melodies is poor, with performance at little better than chance levels for many implant users. This can also be observed when the tunes are familiar and are played as a sequence of isolated notes without accompaniment or harmony. Thus, discrimination of music interval, tone sequences with ascending or descending notes, or simple melodic structures are greatly deteriorated abilities in profound deaf children. On the other hand, one should consider that often the concepts of high and low are difficult even for hearing students who confuse the term with loud and soft. Nevertheless, the ability to differentiate between notes is necessary to study and learn melodies. Perception of timbre, which is usually evaluated by experimental procedures that require subjects to identify musical instrument sounds, is also generally unsatisfactory ${ }^{65}{ }^{68}$. Only the discrimination of the different groups of musical instruments is generally possible (i.e. string-, wood-, brass-, percussion- instruments), while recognising a single instrument among its group is very difficult. Despite all these limitations, Hash ${ }^{68}$ stated, "Considering an 'average' profound hearing loss, the motivated student is capable of learning to play an instrument to at least an intermediate level". Some instruments will be easier for the student who is deaf or hard of hearing to learn, but under the right conditions nearly all are audible over most of their ranges ${ }^{65}{ }^{69}$. Generally, instruments with fixed pitches, such as clarinet, saxophone, flute, piano and organ are recommended. Some instruments produce strong vibration that can be felt on the lips, on the chest or by touching them. Percussion instruments, particularly the bass drum, are great producers of vibration and can therefore provide a very tactile experience. On the other hand, whenever the student is still having difficulties sensing vibrations, instrument selection should include one that is capable of producing sustained rather than percussive sounds, as these may be easier to perceive via residual hearing. The xylophone, with its clear and short sounds, is considered one of the easiest instrument to be perceived by the cochlear implanted child. For further details about the instrument selection for a deaf child, see Hash ${ }^{69}$. Singing activities are also strongly recommended for these children. Cochlear implanted children are generally able to sing familiar songs from memory, though their performance is significantly poorer than in children with normal hearing on almost all pitch-based assessment of singing ${ }^{70}$.

Musical involvement can help a child to develop a positive self-image with opportunities for self-expression and can improve social interactions. Moreover, music can serve as motivational tool for positive behavior and can relieve the tension and struggle that some children experience during language training. The music classroom can play an important role in promoting acceptance and understanding. Musical activity can aid in breaking down any social barriers, thus helping to diminish misconceptions and fear related to hearing loss. 
Darrow ${ }^{64}$ affirms that almost any aural concept can be visually reinforced. The use of Windows Media Player visuals has been recommended because of their colourful representation of music elements such as rhythm, tempo, and melodic direction. In fact, visual representation of music can help to clarify what children perceive. Moreover, body rhythms can be implemented to symbolise rhythmic structure. Pitch can also be illustrated using the body. For example, younger students can crouch down for low notes and stand on their tiptoes with arms above their heads for higher notes.

It has been found that music combined with speech therapy can have positive effects on the development of both good listening habits and auditory skills, and on the development of the suprasegmental elements of language, auditory figure-ground discrimination, sequential memory, voice quality and rhythm of speech ${ }^{6971}$.

Nevertheless, it should be noted that only studies that carried out a long and intensive musical training with deaf children were able to demonstrate a significant benefit on language perception and productions ${ }^{72} 73$. For example, Rouchette et al. ${ }^{74}$ used training that lasted from 1.5 to 4 years conducted by music teachers with a consolidated experience. In contrast, shorter training carried out by parents or caregivers failed to demonstrated significant positive results ${ }^{75}$. Training benefits were evaluated by using the instrument "Sound in Hands" (Fig. 5). Evaluation included four aspects of auditory perception: discrimination, identification, auditory scene analysis and auditory working memory.

In conclusion, it is still debatable whether music training might have a benefit on language perception and production in deaf children. On the other hand, there is a general agreement that musical involvement can help a child to develop a positive self-image with opportunities for selfexpression and can improve social interactions. Moreover, hard of hearing children may enjoy music and motivated students are capable of learning to play an instrument to at least an intermediate level.

\section{Neurofibromatosis 2. Hearing-related management strategies}

Neurofibromatosis 2 (NF2) is a genetic disease (autosomal dominant) with a variable expression due to the different kinds of mutations, mosaicism being the less serious condition. It involves benign tumours of the central nervous system, schwannoma on nerves, or meningiomas. Other tumours are glioma, ependymoma and posterior subcapsular lenticular opacity. Schwannomas on the nerves involve the unavoidable loss of function of the nerve of origin. The incidence is about 1 new case/every year/over 100,000 inhabitants. The mutations are on chromosome 22 in a protein named merlin, which acts as a growth factor regulating (inhibiting) growth.

The crucial aspect of the management of NF2 cases is both the decision of what to do and, not less important, when to

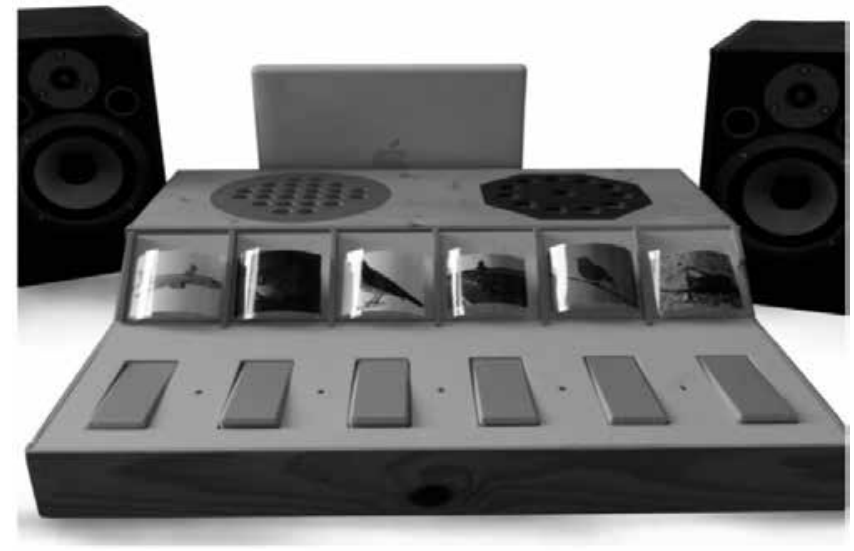

Fig. 5. The instrument "Sound in Hands" designed by McAdams and Bigand in 1993, composed of two speakers, a response platform and a computer.

do something, in the frame of a balance between the natural history of the disease and the consequences of active therapy. The goal of therapy cannot be the cure of the disease, but the setting of pro-active therapy to improve quality of life and prevent the early fate of the disease. When proactive therapy is not successful, functional rehabilitation is feasible, provided early therapy is performed. The role of radiotherapy is limited, for the young age of the patient, the low success of long-term preservation of function and the risk of radio-induced tumours. Clinical observation, radiology and genetics support the diagnosis. The disease is evident in $20 \%$ of cases before 15 years with the bilateral schwannoma, the majority of cases after 18 years presentation ${ }^{76}$.

The new frontiers in the management of NF2 patient account for: 1) the awareness of the importance of early-stage surgery conceived as functional surgery; and 2) the possibility of rehabilitation, especially of the VIII cranial nerve. The most frequent condition in NF2 is bilateral acoustic schwannoma. This involves a relentless growth of the tumour and an unavoidable loss of hearing over the years, which may be progressive or sudden but is the functional endpoint. Tumour growth in NF2 schwannoma is more aggressive and rapid than the sporadic and is often characterised by multiple growing lobules of tumours. The natural history of the tumour is also more associated with facial palsy than in sporadic schwannoma.

The treatment involves two different attitudes: a "conservative" attitude, which means watchful observation of tumour growth, in which treatment is delayed until function is lost (hearing) and tumour growth is no more tolerated due to size and impending risk of brain compression. When surgery is performed, the size of tumour is such to put facial nerve preservation at risk, not differently from what is the rule in the sporadic tumour. Late surgery with a large tumour prevents the possibility of hearing preservation (which is feasible and advisable only at a very early stage of tumour,) and of the cochlear nerve (which may anatomically be preserved only in small-medium size tumours and its possibility of 
functioning with a cochlear implant seems to be inversely related to the preoperative loss of hearing). The advantage of this "conservative" attitude is to leave natural function to patients (hearing and facial nerve) as long as this is possible. The consequences of a delayed surgical treatment are higher intraoperative surgical risks, higher morbidity on the facial nerve and no possibility of hearing preservation/hearing rehabilitation with cochlear implant. Why should we adopt a conservative strategy, which prevents any possibility of preservation/rehabilitation of function? The answer is in the complexity of the disease, where every choice pays a price and has to be balanced very carefully. Sometimes it may be reasonable, for severe NF2 patients, to leave hearing functions intact (on one side at least) as long as this is feasible in the so-called crucial years, when education takes its course and postpone the problem of the management of bilateral acoustic neuroma at a later age. It is evident that this "conservative" attitude, though the most adopted till the 2000's, has limited value nowadays, or rather it is still an option but has to be balanced in the frame of a more updated programme of management of the NF2 patient. The "proactive" attitude involves an early therapy at an early stage tumour. When good function is still present (hearing) it may be preserved with hearing preservation surgery 77 and, if not, may be rehabilitated with cochlear implant ${ }^{78}$. In our experience in sporadic and NF2 acoustic neuromas, we observed better results in hearing rehabilitation with cochlear implant when: 1) the tumour was small and the cochlear nerve did not suffer longtime from the presence of tumour; 2) hearing was preoperatively good and a functioning cochlear nerve could be preserved. The rationale of this proactive surgery is not to rehabilitate function straightforward, but to cure the disease at an early stage with the attempt to preserve natural hearing in the best preoperative conditions and rehabilitate it only in case of failure.
We summarise our guidelines in Table I for sporadic tumours.

In NF2 patients, the choice is more difficult and should be balanced in the frame of the severity of the disease and the expectations of the patient and family.

Some considerations are preliminary to any decisionmaking process in the NF2 patient.

A multidisciplinary nature of the approach (neuro-otologist, neurosurgery, genetics, radiotherapy, oncologist); the high rates of success of hearing preservation surgery in sporadic acoustic neuroma are probably less reproducible in the NF2 patient ${ }^{69}$. The inexorable growth, the multilobular pattern of presentation of the tumour relate to more aggressive involvement of the cochlear nerve and, to a lesser extent, of the facial nerve; bilateral acoustic neuroma is often not an isolated condition, but in the NF2 patient it is associated with other sites as meningioma, schwannoma, gliomas. Surgery may involve higher morbidity due to the presence of other adjacent tumours (i.e. lower posterior fossa, jugular foramen, foramen magnum) that may affect the function of other cranial nerves and the CSF pathways; hearing rehabilitation with $\mathrm{ABI}$, though always feasible, should be the last option after any attempt of hearing preservation/hearing rehabilitation with cochlear implant. Similarly, good facial nerve preservation should be the goal in small-medium size tumours. When not feasible, intraoperative reconstruction of the facial nerve with a graft should be the option.

In conclusion, the new trend in NF2 management involves proactive therapy in the attempt to preserve function or rehabilitate it in case of failure. The attitude of leaving the disease at its fate leads to the unavoidable condition where it is too late to spare function or rehabilitate it. The crucial step of when active therapy should begin to interfere with the natural course of the disease is the main aspect of any choice, as well

Table I. Treatment algorithm of sporadic vestibular schwannoma in our centre.

\begin{tabular}{|c|c|c|}
\hline T size $(\mathrm{mm})$ & Decision factors & Treatment \\
\hline \multirow[t]{3}{*}{$\begin{array}{l}\text { Intrameatal or } \\
\mathrm{T}<10 \text { in } \mathrm{CPA}\end{array}$} & Good hearing class A (B) & $\begin{array}{l}\text { Hearing preservation surgery } \\
\text { (observation) }\end{array}$ \\
\hline & Good hearing class $A(B)+$ risk & Observation* \\
\hline & Bad hearing & Observation* or hearing rehabilitation \\
\hline \multirow[t]{3}{*}{ T 10-15 } & No growth & Observation* or surgery \\
\hline & Growth & Surgery \\
\hline & Growth + risk & Radiotherapy \\
\hline T 15-25 & - & Surgery or Radiotherapy \\
\hline \multirow[t]{2}{*}{$T>25$} & - & Surgery \\
\hline & Risk & Partial surgery + radiotherapy \\
\hline Any size & Cystic tumour & Surgery \\
\hline
\end{tabular}

\footnotetext{
${ }^{*}$ Active treatment is planned in case of vertigo or new onset of 7 the c.n. defect or C.I. rehabilitation.
} 
as the awareness of the updated surgical procedures of sparing function and rehabilitate it, such as in bilateral schwannoma. When the tumours affect the mixed nerves (i.e. in the jugular foramen, IX-XII cranial nerves), preservation or surgical rehabilitation of function is, at present, still not feasible.

\section{Conclusions}

Rehabilitation programs for paediatric patients with disorders of voice, speech and language, communication and hearing, deglutition and breathing are not widely available in either hospital settings or in long-term care facilities. In most countries, the number of physicians and technicians is still inadequate.

The goal for the future will be establishment of highly specialised third level centres, where multidisciplinary teams dedicated to paediatric patients can collaborate.

\section{Acknowledgments}

Thanks to all the authors and coauthors of the Official Report of XXIII Conference of Italian Society of Pediatric Otorhinolaryngology (Rome, November, 05-07th 2015) for their meaningful contributions: Claudia Aimoni; Roberto Albera; Paolo Aluffi Valletti, Pasqualina Apisa; Gennaro Auletta; Cristiano Balzanelli; Eugenio Baraldi; Maria Rosaria Barillari; Umberto Barillari; Giovanni Bastanza; Stefano Berettini; Daniele Borsetto; Sergio Bottero; Elona Cama; Elena Cantone; Eleonora Capolongo; Federico Caranzano; Laura Carrabba; Claudia Cassandro; Alessandro Castiglione; Michele Cavaliere; Antonella Cerchiari; Francesca Cianfrone; Giancarlo Cianfrone; Andrea Ciorba; Guido Conti; Virginia Corazzi; Erika Crosetti; Domenico Cuda; Cesare Cutrone; Concetta D'adamo; Wladimiro De Colle;-Cosimo De Filippis; Gessica Della Bella; Antonio Della Volpe; Antonietta De Lucia; Vincenzo De Maio; Giovanni Carlo De Vincentiis; Marco De Vincentiis; Andrea De Vito; Daniele Farneti; Anna Rita Fetoni; Francesca Forli; Andrea Franchella; Sebastiano Franchella; Annamaria Franze'; Stefano Fusetti; Franco Fussi; Giovanna Gaggero; Samanta Gallo; Roberto Gallus; Elisabetta Genovese; Sara Giannantonio; Daniela Ginocchio; Pamela Giordano; Marta Gisolo; Cristina Gondiu; Massimo Grassi; Francesca Graziani; Antonio Greco; Maria Consolazione Guarnaccia; Maurizio Iengo; Alessandro Incognito; Sabina Iozzino; Carla Laria; Paola Leone; Marco Lionello; Lucia Lora; Rita Malesci; Nicola Mansi; Raffaella Marchi; Giulia Marini; Pasquale Marsella; Antonio Mazzoni; Massimo Mesolella; Carla Morando; Laura Moschino; Francesco Mozzanica; Alessandra Murri; Enrico Muzzi; Flavia Nardi; Cristina Nicole'; Ciro Niri; Eva Orzan; Concettina Pacifico; Silvia Palma; Gaetano Paludetti; Annalisa Panarese; Maria Laura Panatta; Claudio Parrilla; Desiderio Passali; Francesco Maria Passali; Sara Penco; Francesco Pia; Alessandro Piacente; Pasqualina Maria Picciotti; Federica Pizzoli; Luca
Oscar Redaelli De Zinis; Andrea Ricci Maccarini; Lara Righetto; Sergio Ronfini; Gianni Ruoppolo; Roberto Saetti; Rosamaria Santarelli; Athanasios Saratziotis; Marianna Sari; Letizia Scarponi; Franco Schiavi; Antonio Schindler; Emanuele Scarano; Alessandro Scorpecci; Pietro Scimemi; Marina Silvestrini; Emanuela Sitzia; Lucrezia Spadera; Irma Spahiu; Giacomo Spinato; Roberto Spinato; Giovanni Succo; Fausto Taranto; Valeria Testugini; Stefano Tiglie'; Giancarlo Tirelli; Rosaria Turchetta; Laura Travan; Maria Luisa Tropiano; Marilena Trozzi; Alfonso M.Varricchio; Irene Vernero.

\section{References}

1 Sobol SE, Samadi DS, Kazahaya K, et al. Trends in the management of pediatric chronic sinusitis: survey of the American Society of Pediatric Otolaryngology. Laryngoscope 2005;115:78-80.

2 Fokkens WJ, Lund VJ, Mullol J, et al. EPOS 2012: European position paper on rhinosinusitis and nasal polyps. A summary for otorhinolaryngologists. Rhinology 2012;50:1-12.

3 Cunningham MJ, Chiu EJ, Landgraf JM, et al. The health impact of chronic recurrent rhinosinusitis in children. Arch Otolaryngol Head Neck Surg 2000;126:1363-8.

4 Terrell AM, Ramadan HH. Correlation between $\mathrm{SN}-5$ and computed tomography in children with chronic rhinosinusitis. Laryngoscope 2009;119:1394-8.

5 Corrales CE, Koltai PJ. Choanal atresia: current concepts and controversies. Curr Opin Otolaryngol Head Neck Surg 2009;17:466-70.

6 Friedman NR, Mitchell RB, Bailey CM, et al. Management and outcome of choanal atresia correction. Int $\mathrm{J}$ Pediatr Otorhinolaryngol 2000;52:45-51.

7 Saetti R, Emanuelli E, Cutrone C, et al. The treatment of choanal atresia. Acta Otorhinolaryngol Ital 1998;18:307-12.

8 Pirsig W. Surgery of choanal atresia in infants and children: historical notes and updated review. Int J Pediatr Otorhinolaryngol 1986;11:153-70.

9 Cedin AC, Atallah AN, Andriolo RB, et al. Surgery for congenital choanal atresia. Cochrane Database Syst Rev 2012;2:CD008993.

10 Bedwell JR, Choi SS. Are stents necessary after choanal atresia repair? Laryngoscope 2012;122:2365-6.

11 Zuckerman JD, Zapata S, Sobol SE. Single-stage choanal atresia repair in the neonate. Arch Otolaryngol Head Neck Surg 2008;134:1090-3.

12 Ibrahim AA, Magdy EA, Hassab MH. Endoscopic choanoplasty without stenting for congenital choanal atresia repair. Int J Pediatr Otorhinolaryngol 2010;74:144-50.

13 Strychowsky JE, Kawai K, Moritz E, et al. To stent or not to stent? A meta-analysis of endonasal congenital bilateral choanal atresia repair. Laryngoscope 2015;126:21827.

14 Teissier N, Kaguelidou F, Couloigner V, et al. Predictive factors for success after transnasal endoscopic treatment of choanal atresia. Arch Otolaryngol Head Neck Surg 2008;134:57-61. 
15 Carroll JL, McColley SA, Marcus CL, et al. Inability of clinical history to distinguish primary snoring from obstructive sleep apnea syndrome in children. Chest 1995;108:610-8.

16 Piumetto E, Sammartano AM, Meinardi G, et al. Diagnostic and therapeutic iter in paediatric OSAS: personal experience. Acta Otorhinolaryngol Ital 2011;31:149-53.

${ }^{17}$ Friedman M, Wilson M, Lin HC, et al. Updated systematic review of tonsillectomy and adenoidectomy for treatment of pediatric obstructive sleep apnea/hypopnea syndrome. Otolaryngol Head Neck Surg 2009;140:800-8.

18 Stewart MG, Glaze DG, Friedman EM, et al. Quality of life and sleep study findings after adenotonsillectomy in children with obstructive sleep apnea. Arch Otolaryngol Head Neck Surg 2005;131:308-14.

19 Albera R, Piumetto E, Gervasio FC, et al. Conseguenze dell'OSAS pediatrica in ambito foniatrico-logopedico. In: Relazione Ufficiale XXII Congresso Nazionale SIOP. Alba, Sett 2013.

20 Moeller JL Orofacial myofunctional therapy: why now? Cranio 2012;30:235-6.

21 Cerchiari A, Falbo M. Il trattamento riabilitativo miofunzionale dell'OSAS. In: Relazione Ufficiale XXII Congresso Nazionale SIOP. Alba, Sett 2013.

22 Monnier P. Pediatric Airway Surgery. Berlin Heidelberg: Springer-Verlag; 2011. p. 107-117.

23 Saetti R, Silvestrini M, Galiotto M, et al. Contact laser surgery in treatment of vocal fold paralysis. Acta Otorhinolaryngol Ital 2003;23:33-7.

24 Schwartz SR, Cohen SM, Dailey SH, et al. Clinical practice guideline: hoarseness (dysphonia). Otolaryngol Head Neck Surg 2009; 141:S1-S31.

25 Preuss SF, Klussmann JP, Jungehulsing M, et al. Long-term results of surgical treatment for recurrent respiratory papillomatosis. Acta Otolaryngol 2007;127:1196-201.

26 Ilmarinen T, Nissilä H, Rihkanen $\mathrm{H}$, et al. Clinical features, health-related quality of life, and adult voice in juvenileonset recurrent respiratory papillomatosis. Laryngoscope 2011;121:846-51.

27 Fusconi M, Grasso M, Greco A, et al. Recurrent respiratory papillomatosis by HPV: review of the literature and update on the use of cidofovir. Acta Otorhinolaryngol Ital 2014;34:375-81.

28 Holler T, Allegro J, Chadha NK, et al. Voice outcomes following repeated surgical resection of laryngeal papillomata in children. Otolaryngol Head Neck Surg 2009;141:522-6.

29 Holland BW, Koufman JA, Postma GN, et al. Laryngopharyngeal reflux and laryngeal web formation in patients with pediatric recurrent respiratory papillomas. Laryngoscope 2002;112:1926-9.

30 Ida JB, Thompson DM. Pediatric stridor. Otolaryngol Clin North Am 2014;47:795-819.

31 Giannoni C, Sulek M, Friedman EM, et al. Gastroesophageal reflux association with laryngomalacia: a prospective study. Int J Pediatr Otorhinolaryngol 1998;43:11-20.

32 Cotton RT, Prescott CA. Congenital anomalies of the larynx. Practical Pediatric Otolaryngology. Philadelphia/New York: Lippincott-Raven; 1999. p.497-514.

33 Butnaru CS, Colreavy MP, Ayari S, et al. Tracheotomy in chil- dren: evolution in indications. Int J Pediatr Otorhinolaryngol 2006;70:115-9.

34 Durbin CG Jr. Tracheostomy: why, when and how? Respir Care 2010;55:1056-68.

35 Ciaglia P, Firsching R, Syniec C. Elective percutaneous dilatational tracheostomy. A new simple bedside procedure; preliminary report. Chest 1985;87:715-9.

${ }^{36}$ Mitchell RB, Hussey HM, Setzen G, et al. Clinical consensus statement: tracheostomy care. Otolaryngol Head Neck Surg 2013;148:6-20.

37 National Guidelines Clearinghouse. Best evidence statement (BESt). Basic Pediatric Tracheostomy Care; 2011.

38 Cincinnati Children's Hospital Medical Center: Best evidence statement (BESt). Basic pediatric tracheostomy care. Cincinnati $(\mathrm{OH})$ : Cincinnati Children's Hospital Medical Center; 2011.

39 Curtis DJ, Hudson T. Laryngotracheal aspiration: analysis of specific neuromuscular factors. Radiology 1983;149:517-22.

40 Curtis DJ, Sepulveda GU. Epiglottic motion: video recording of muscular dysfunction. Radiology 1983;148:473-7.

${ }^{41}$ Miller CK, Willging JP. The implications of upper-airway obstruction and successful infant feeding. Semin Speech Lang 2007;28:190-203.

42 Williams S. Pediatric feeding and swallowing problems: an interdisciplinary team approach. Perspectives in practice. Can J Diet Pract Res 2006;67:185-90.

43 Willing PJ. Benefit of feeding assessment before pediatric airway reconstruction. Laryngoscope 2000;110:826-34.

44 Smith LP. Management of oral feeding in children undergoing airway reconstruction. Laryngoscope 2009;119:967-73.

45 Andreoli SM. Feeding status after pediatric laryngotracheal reconstruction. Otolaryngol Head Neck Surg 2010;143:210-3.

46 Estill J. The primer of compulsory figures. www.evts.com

47 Fussi F, Turlà E. Il trattamento delle disfonie. Una prospettiva per il metodo Estill Voicecraft. Torino: Omega Ed.; 2008

48 Borragan A, Lucchini E, Agudo M, et al. Il Metodo Propriocettivo Elastico (PROEL) nella terapia vocale. Acta Phoniatrica Latina 2008;30:17-50.

49 De Maio V, Mansi N, Capolongo E, et al. La riabilitazione delle disfonie organiche infantili con il metodo propriocettivo-elastico adattato ai bambini. In: Martini A, Trevisi P, editors. La riabilitazione in ORL Pediatrica. Torino: Omega Ed.; 2015. p. 195-200.

50 Schindler A, Tiddia C, Ghidelli C, et al. Adaptation and validation of the Italian Pediatric Voice Handicap Index. Folia Phoniatr Logop 2011;63:9-14.

51 Ricci G, Della Volpe A, Faralli M, et al. Results and complications of the Baha system (bone-anchored hearing aid). Eur Arch Otorhinolaryngol 2010;267:1539-45.

52 Bovo R. Simplified technique without skin flap for the boneanchored hearing aid (BAHA) implant. Acta Otorhinolaryngol Ital 2008;28:252-5.

53 Shonka DC Jr, Livingston WJ 3rd, Kesser BW. The Jahrsdoerfer grading scale in surgery to repair congenital aural atresia. Arch Otolaryngol Head Neck Surg 2008;134:873-7. 
54 Evans AK, Kazahaya K. Canal atresia: "surgery or implantable hearing devices? The expert's question is revisited". Int J Pediatr Otorhinolaryngol 2007;71:367-74.

55 Bouhabel S, Arcand P, Saliba I. Congenital aural atresia: boneanchored hearing aid vs. external auditory canal reconstruction. Int J Pediatr Otorhinolaryngol 2012;76:272-7.

56 Nadaraja GS, Gurgel RK, Kim J, et al. Hearing outcomes of atresia surgery versus osseointegrated bone conduction device in patients with congenital aural atresia: a systematic review. Otol Neurotol 2013;34:1394-9.

57 Bovo R, Martini A, Agnoletto M, et al. Auditory and academic performance of children with unilateral hearing loss. Scand Audiol Suppl 1988;30:71-4.

58 Baguley DM, Bird J, Humphriss RL, et al. The evidence base for the application of contralateral bone anchored hearing aids in acquired unilateral sensorineural hearing loss in adults. Clin Otolaryngol 2006;31:6-14.

59 Paludetti G, Conti G, DI Nardo W, et al. Infant hearing loss: from diagnosis to therapy. Official Report of XXI Conference of Italian Society of Pediatric Otorhinolaryngology. Acta Otorhinolaryngol Ital 2012;32:347-70.

60 Ciorba A, Bovo R, Trevisi P, et al. Postoperative complications in cochlear implants: a retrospective analysis of 438 consecutive cases. Eur Arch Otorhinolaryngol 2012;269:1599-603.

61 Bovo R, Trevisi P, Ghiselli $\mathrm{S}$, et al. Is very early hearing assessment always reliable in selecting patients for cochlear implants? A case series study. Int J Pediatr Otorhinolaryngol 2015;79:25-31.

62 Trevisi P, Ciorba A, Aimoni C, et al. Outcomes of long-term audiological rehabilitation in charge syndrome. Acta Otorhinolaryngol Ital 2016;36:206-14.

63 Ford, T. The effect of musical experiences and age on the ability of deaf children to discriminate pitch of complex tones. (Doctoral dissertation, The University of North Carolina at Greensboro 1985) Dissertation Abstracts International 47 (09A), 2921.

${ }^{64}$ Darrow AA. Sounds in silence. Research in music and deafness. Update Application of Research in Music Education 2006;25:5-16.

${ }_{65}$ Gfeller K. Music-based training for pediatric CI recipients: A systematic analysis of published studies. Eur Ann Otorhinolaryngol Head Neck Dis 2016;133(Suppl 1):S50-6.
66 Schraer-Joiner L, Chen-Hafteck L. The responses of preschoolers with cochlear implants to musical activities: a multiple case study. Early Child Development and Care 2009;179:785-98.

67 Vongpaisal T, Trehub SE, Schellenberg EG. Song recognition by children and adolescents with cochlear implants. J Speech Lang Hear Res 2006;49:1091-103.

68 Hash PM. Teaching Instrumental music to deaf and hard of hearing students. Research and Issues in Music Education 2003; http://files.eric.ed.gov/fulltext/EJ852403.pdf.

69 Hash PM. Instrument Selection. Research and Issues in $\mathrm{Mu}-$ sical Education 2003. www.stthomas.edu/rimeonline.

$70 \mathrm{Xu} \mathrm{L}$, Zhou N, Chen X, et al. Vocal singing by prelingually-deafened children with cochlear implants. Hear Res 2009;255:129-34.

71 Silvestre N, Valero J. Oral language acquisition by deaf Pupils in primary education: impact of musical education. Eur J Special Needs Educ 2005;20:195-213.

72 Torppa R, Faulkner A, Huotilainen M, et al. The perception of prosody and associated auditory cues in early-implanted children: the role of auditory working memory and musical activities. Int J Audiol 2014;53:182-91.

73 Di Nardo W, Schinaia L, Anzivino R, et al. Musical training software for children with cochlear implants. Acta Otorhinolaryngol Ital 2015;35:249-57.

74 Rochette F, Moussard A, Bigand E. Music lessons improve auditory perceptual and cognitive performance in deaf children. Front Hum Neurosci 2014;8:488.

75 Yucel E, Sennaroglu G, Belgin E. The family oriented musical training for children with cochlear implants: speech and musical perception results of two years follow-up. Int J Pediatr Otorhinolaryngol 2009;73:1043-52.

76 Ahn MS, Jackler RK, Lustig LR. The early history of the neurofibromatosis. Evolution of the concept of neurofibromatosis type 2. Arch Otolaryngol Head Neck Surg 1996;122:1240-9.

77 Mazzoni A, Biroli F, Foresti C, et al. Hearing preservation surgery in acoustic neuroma. Slow progress and new strategies. Acta Otorhinolaryngol Ital 2011;31:76-84.

78 Lustig LR, Yeagle J, Driscoll CL, et al. Cochlear implantation in patients with neurofibromatosis type 2 and bilateral vestibular schwannoma. Otol Neurotol 2006;27:512-8. 